\title{
Treatment of MOG-IgG-associated disorder with rituximab: An international study of 121 patients
}

\author{
Whittam, Daniel H ; Cobo-Calvo, Alvaro ; Lopez-Chiriboga, A Sebastian ; Pardo, Santiago ; Gornall, \\ Matthew ; Cicconi, Silvia ; et al ; Jelcic, Ilijas
}

\begin{abstract}
OBJECTIVE To assess the effect of anti-CD20 B-cell depletion with rituximab (RTX) on relapse rates in myelin oligodendrocyte glycoprotein antibody-associated disorder (MOGAD). METHODS Retrospective review of RTX-treated MOGAD patients from 29 centres in 13 countries. The primary outcome measure was change in relapse rate after starting rituximab (Poisson regression model). RESULTS Data on 121 patients were analysed, including 30 (24.8\%) children. Twenty/121 (16.5\%) were treated after one attack, of whom 14/20 (70.0\%) remained relapse-free after median (IQR) 11.2 (6.3-14.1) months. The remainder (101/121, 83.5\%) were treated after two or more attacks, of whom 53/101 (52.5\%) remained relapse-free after median 12.1 (6.3-24.9) months. In this 'relapsing group', relapse rate declined by $37 \%(95 \% \mathrm{CI}=19-52 \%, \mathrm{p}<0.001)$ overall, $63 \%(95 \% \mathrm{CI}=35-79 \%, \mathrm{p}=0.001)$ when RTX was used first line $(\mathrm{n}=47)$, and $26 \%(95 \% \mathrm{CI}=2-44 \%, \mathrm{p}=0.038)$ when used after other steroid-sparing immunotherapies $(\mathrm{n}=54)$. Predicted 1-year and 2-year relapse-free survival was $79 \%$ and $55 \%$ for first-line RTX therapy, and $38 \%$ and $18 \%$ for second-/third-line therapy. Circulating CD $19^{+}$B-cells were suppressed to $<1 \%$ of total circulating lymphocyte population at the time of 45/57 (78.9\%) relapses. CONCLUSION RTX reduced relapse rates in MOGAD. However, many patients continued to relapse despite apparent B-cell depletion. Prospective controlled studies are needed to validate these results.
\end{abstract}

DOI: https://doi.org/10.1016/j.msard.2020.102251

Posted at the Zurich Open Repository and Archive, University of Zurich ZORA URL: https://doi.org/10.5167/uzh-192579

Journal Article

Accepted Version

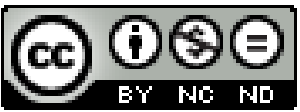

The following work is licensed under a Creative Commons: Attribution-NonCommercial-NoDerivatives 4.0 International (CC BY-NC-ND 4.0) License.

Originally published at:

Whittam, Daniel H; Cobo-Calvo, Alvaro; Lopez-Chiriboga, A Sebastian; Pardo, Santiago; Gornall, Matthew; Cicconi, Silvia; et al; Jelcic, Ilijas (2020). Treatment of MOG-IgG-associated disorder with rituximab: An international study of 121 patients. Multiple Sclerosis and Related Disorders, 44:102251. DOI: https://doi.org/10.1016/j.msard.2020.102251 


\section{Treatment of MOG-IgG-associated demyelinating disorder with rituximab: a multinational retrospective study of 121 patients.}

Daniel Whittam ${ }^{1}$, Alvaro Cobo-Calvo ${ }^{2}$, A. Sebastian Alfonzo Lopez-Chiriboga ${ }^{3}$, Santiago Pardo $^{4}$, Matthew Gornall ${ }^{5}$, Silvia Cicconi ${ }^{5}$, James Dodd ${ }^{5}$, Alexander Brandt ${ }^{6}$, Klaus Berek $^{7}$, Thomas Berger ${ }^{7}$, Ilijas Jelcic ${ }^{8}$, Grace Gombolay ${ }^{9}$, Luana Micheli Oliveira ${ }^{10}$, Dagoberto Callegaro $^{10}$, Kimihiko Kaneko ${ }^{11}$, Tatsuro Misu ${ }^{11}$, Marco Capobianco ${ }^{12}$, Bruno Brochet ${ }^{13}$, Bertrand Audoin ${ }^{14}$, Guillaume Mathey ${ }^{15}$, David Laplaud ${ }^{16}$, Eric Thouvenot ${ }^{17}$, Mikaël Cohen $^{18}$, Ayman Tourbah ${ }^{19}$, Elisabeth Maillart ${ }^{20}$, Jonathan Ciron ${ }^{21}$, Romain Deschamps ${ }^{22}$, Damien Biotti ${ }^{23}$, Kevin Rostasy ${ }^{24}$, Rinze Neuteboom ${ }^{25,26}$, Cheryl Hemingway ${ }^{27}$, Rob Forsyth $^{28}$, Marcelo Matiello ${ }^{9}$, Stewart Webb ${ }^{29}$, David Hunt ${ }^{30}$, Katy Murray ${ }^{30}$, Yael Hacohen $^{27}$, Ming Lim ${ }^{31,32}$, Isobel Leite ${ }^{33}$, Jacqueline Palace ${ }^{33}$, Andreas Lutterotti ${ }^{8}$, Kazuo Fujihara $^{11}$, Ichiro Nakashima ${ }^{11}$, Jeffrey L. Bennett ${ }^{34}$, Lekha Pandit ${ }^{35}$, Tanuja Chitnis ${ }^{9}$, Brian Weinshenker ${ }^{3}$, Brigitte Wildemann ${ }^{36}$, Douglas Kazutoshi Sato ${ }^{10,11,37}$, Su-Hyun Kim ${ }^{38}$, Saif Huda $^{1}$, Ho Jin Kim ${ }^{38}$, Markus Reindl ${ }^{7}$, Michael Levy ${ }^{4}$, Sven Jarius ${ }^{36}$, Silvia Tenembaum ${ }^{39}$, Friedemann Paul ${ }^{6}$, Sean Pittock ${ }^{3}$, Romain Marignier ${ }^{2}$, Anu Jacob ${ }^{1,5}$.

${ }^{1}$ Department of Neurology, The Walton Centre NHS Foundation Trust, Lower Lane, Liverpool, $U K$.

${ }^{2}$ Hôpital Neurologique Pierre Wertheimer Hospices Civils de Lyon, Lyon, France.

${ }^{3}$ Department of Neurology, Mayo Clinic, Rochester, Minnesota, USA.

${ }^{4}$ Department of Neurology, Massachusetts General Hospital and Harvard Medical School, Boston, MA, USA.

${ }^{5}$ The University of Liverpool, Liverpool, UK.

${ }^{6}$ Charité Universitätsmedizin Berlin, Berlin, Germany. 
${ }^{7}$ Medical University of Innsbruck, Innsbruck, Austria.

${ }^{8}$ UniversitätsSpital Zürich, Zürich, Switzerland.

${ }^{9}$ Massachusetts General Hospital, Harvard University, Boston, Massachusetts, USA.

${ }^{10}$ Hospital das Clinicas, Faculty of Medicine, University of Sao Paulo, Sao Paulo, Brazil.

${ }^{11}$ Tohoku University Graduate School of Medicine, Sendai, Japan.

${ }^{12}$ Neurology Dept. - Regional MS Centre, S. Luigi University Hospital, Orbassano, Italy.

${ }^{13}$ CHU de Bordeaux \& INSERM U 1215, University of Bordeaux, Bordeaux, France.

${ }^{14}$ Aix Marseille Univ, APHM, Hôpital de La Timone, Marseille, France.

${ }^{15}$ Nancy University Hospital and Inserm CIC 1433, Nancy, France.

${ }^{16}$ Nantes University Hospital, Nantes, France.

${ }^{17}$ Hôpital Carémeau, Nimes University Hospital, Nimes, France.

${ }^{18}$ Université Côte d'Azur, Centre Hospitalier Universitaire de Nice, Nice, France.

${ }^{19}$ Reims University Hospital, University of Reims Champagne-Ardenne, Reims, and LPN EA 2027, University of Paris VIII, Saint-Denis, France.

${ }^{20}$ Pitié-Salpêtrière Hospital, APHP, Paris, France.

${ }^{21}$ Poitiers University Hospital, Poitiers, France.

${ }^{22}$ Fondation A. De Rothschild, Paris, France.

${ }^{23}$ University Hospital of Purpan, Toulouse, France.

${ }^{24}$ Department of Pediatric Neurology, Children's Hospital Datteln, University

Witten/Herdecke, Witten, Germany

${ }^{25}$ Department of Paediatric Neurology, Erasmus MC-Sophia, Rotterdam, The Netherlands

${ }^{26}$ Department of Neurology, MS Centre ErasMS, Erasmus MC, Rotterdam, The Netherlands

${ }^{27}$ Department of Paediatric Neurology, Great Ormond Street Hospital for Children, London, $U K$

${ }^{28}$ Institute of Neuroscience, Newcastle University, UK. 
${ }^{29}$ Department of Neurology, Queen Elizabeth University Hospital, Glasgow, UK.

${ }^{30}$ Anne Rowling Regenerative Neurology Clinic, University of Edinburgh, Edinburgh, UK.

${ }^{31}$ Children's Neuroscience, Evelina London Children's Hospital @ Guy’s and St Thomas' NHS Foundation Trust, London, UK.

${ }^{32}$ Faculty of Life Sciences and Medicine, King's College London, London, UK.

${ }^{33}$ Nuffield Department of Clinical Neurosciences, John Radcliffe Hospital, Oxford, UK.

${ }^{34}$ Departments of Neurology and Ophthalmology, Program in Neuroscience, University of Colorado School of Medicine, Aurora, Colorado, USA.

${ }^{35}$ Nitte University, Mangaluru, Karnataka, India.

${ }^{36}$ Molecular Neuroimmunology Group, Department of Neurology, University of Heidelberg, Heidelberg, Germany.

${ }^{37}$ Pontificia Universidade Catolica Rio Grande Do Sul, Porto Alegre RS, Brazil.

${ }^{38}$ Research Institute and Hospital of National Cancer Center, Goyang, Korea.

${ }^{39}$ National Pediatric Hospital Dr. Juan P. Garrahan, Ciudad Autónoma de Buenos Aires, Argentina.

\begin{abstract}
Introduction: Rituximab (RTX), a B-cell depleting monoclonal antibody, is effective in treating many autoimmune disorders, including multiple sclerosis and aquaporin-4 antibody positive neuromyelitis optica spectrum disorders (AQP4-IgG-positive NMOSD). Its efficacy in a large cohort of patients with myelin oligodendrocyte glycoprotein antibody-associated disorder (MOGAD) has not been assessed.
\end{abstract}


Methods: Centres around the world with expertise in treating MOGAD were invited to provide retrospective data on relapses and disability in all of their MOGAD patients treated with RTX. Data were collected on 132 patients from 29 centres in 13 countries.

Results: Data on 121 patients were analysed; 103/121 (85.1\%) were white and 30/121 $(24.8 \%)$ were children. Because relapse risk after initial attack is uncertain in MOGAD, we analysed patients in two groups: those treated with RTX after a single attack, and those treated after two or more attacks (established relapsing disorders). 20/121 (16.5\%) were treated after a single attack, of whom 14/20 (70.0\%) remained relapse-free at median followup of 11.2 months. $6 / 20$ relapsed after a median interval of 2.6 months. 101/121 (83.5\%) were treated with RTX after two or more attacks, and a 37\% $(95 \% \mathrm{CI}=19-52 \%, \mathrm{p}<0.001)$ reduction in relapse rate was observed overall. In this group, RTX was used as a $1^{\text {st }}$ line steroid-sparing immunotherapy in $47 / 101(46.5 \%)$ and as a $2^{\text {nd }} / 3^{\text {rd }}$ line therapy in in $54 / 101$ (53.5\%), most commonly after mycophenolate mofetil or azathioprine. The reduction in relapse rate with $1^{\text {st }}$ line $\mathrm{RTX}$ therapy was $63 \%(95 \% \mathrm{CI}=35-79 \%, \mathrm{p}=0.001)$, and for $2^{\text {nd }} / 3^{\text {rd }}$ line therapy $26 \%(95 \% \mathrm{CI}=2-44 \%, \mathrm{p}=0.038)$. In the relapsing group the predicted relapse-free survival at 2 years on RTX was $55 \%$ for $1^{\text {st }}$ line therapy, and $18 \%$ for $2^{\text {nd }} / 3^{\text {rd }}$ line therapy. Circulating $\mathrm{CD}_{1} 9^{+} \mathrm{B}$-cells were undetectable or suppressed to $<1 \%$ of total lymphocyte population at the time of 45/57 (78.9\%) relapses. Median EDSS scores improved, and visual acuities did not change on RTX, though these data were incomplete.

Conclusion: RTX treatment reduced relapse rates in MOGAD. However a significant proportion of patients continued to relapse, particularly those refractory to other non-steroid immunotherapies. Many relapses occurred despite robust B-cell depletion. The reduction in 
relapse rates in this study is lower than that reported in AQP4-IgG-positive NMOSD. Further studies are therefore needed to inform the management of MOGAD.

\section{Introduction}

Myelin oligodendrocyte glycoprotein (MOG) is expressed on the outermost surface of myelin in the central nervous system and is speculated to provide structural integrity to the myelin sheath. Autoantibodies of the immunoglobulin G1 subtype targeting full-length human MOG (MOG-IgG) have been recently identified in the sera of both children and adults with a CNS inflammatory disease that is distinct from multiple sclerosis (MS) ${ }^{1-3}$ MOG-IgG may be detected in patients presenting with optic neuritis (ON), transverse myelitis (TM), acute disseminated encephalomyelitis (ADEM), encephalitis (including those with seizures) and rhombencephalitis. $^{4-6} 30-80 \%$ of patients relapse after an initial attack, ${ }^{6-9}$ and some fulfil revised diagnostic criteria for aquaporin-4 antibody (AQP4-IgG)-negative neuromyelitis optica spectrum disorders (NMOSD). ${ }^{9,10}$

Although MOG-IgG-associated disorder (MOGAD) may overall be less severe than AQP4IgG-positive NMOSD, a significant proportion of patients do experience relapses and may accrue significant disability; severe visual impairment occurred in $36 \%$ of one cohort. ${ }^{9}$ Longterm immunomodulatory treatment may therefore be reasonable in relapsing patients, especially those with severe attacks. However, there is very limited data on natural history, prognostic markers and treatment responses in MOGAD to inform the optimal treatment strategy. Drugs and treatment algorithms have been adopted from those being used in NMOSD and ADEM, and include corticosteroids, intravenous immunoglobulin (IVIg), azathioprine (AZA), mycophenolate mofetil (MMF) and rituximab (RTX). ${ }^{11-13}$ The few retrospective studies to date suggest a benefit of these immunotherapies, but the numbers of 
patients treated with individual medications are relatively small and these studies do not provide a robust comparison between therapies..$^{7-9,14,15}$

RTX is a chimeric monoclonal antibody directed against CD20, a B-cell surface marker. Following intravenous infusion, circulating B-cells are rapidly eliminated, often to undetectable levels, an effect that persists for 6-12 months on average. Further infusions can be given at fixed six-month intervals or timed according to B-cell/memory B-cell repopulation.

Anti-CD20 B-cell depletion has proven effective in MS. ${ }^{16-19}$ Similarly, numerous, predominantly retrospective, observational studies in AQP4-IgG-positive NMOSD have consistently reported approximately $80 \%$ reductions in annualised relapse rates following initiation of RTX. ${ }^{20-29}$ Given its efficacy in similar neuroinflammatory disorders, it is hoped that RTX may also be effective in MOGAD, but its benefit is not yet defined.

\section{Aim}

To examine the efficacy of RTX in a large international cohort of patients with MOGAD

\section{Methods}

Two authors (DHW and AJ) identified 24 neurologists and paediatric neurologists around the world with an interest in antibody-mediated CNS inflammation, 17 of whom $(70.8 \%)$ submitted demographic, relapse and disability data on all patients in their care meeting the study inclusion criteria (box 1). They also provided the total number of MOGAD patients under their care. 


\section{Box 1: Study inclusion criteria}

1. At least one clinical and MRI-confirmed event consistent with CNS inflammation.

2. MOG-IgG positive by live or fixed cell-based assay incorporating an IgGspecific secondary antibody (MOG-IgG ELISA was not included).

3. AQP4-IgG negative by live or fixed cell-based assay.

4. Treatment with RTX at any point.

We included patients on 'fixed' RTX regimens, as long as the dosing interval did not exceed 6-months, and patients on variable dosing regimens, provided they had adhered to at least two-monthly monitoring of either $\mathrm{CD} 19^{+} \mathrm{B}$-cells or $\mathrm{CD} 19^{+} / \mathrm{CD} 27^{+}$memory B-cells in peripheral circulation.

Relapses were based on the judgement of the investigators at each participating centre. In clinical practice, relapses are defined as a new or worsening symptomatic presentation, supported by a change in neurological examination, and confirmed by MRI as necessary. We asked investigators to provide $\mathrm{CD} 19^{+} \mathrm{B}$-cell counts at the time of relapses if available.

We analysed treatment effect in several ways: A Poisson regression model was fitted to the data, with a random effect by patient level, to compare the relapse rate before and after initiating RTX (onset attack is excluded from this model). We also calculated the change in median annualised relapse rate (ARR) after initiation of RTX, and used Kaplan-Meier survival curves to estimate relapse-free survival. ARR is defined as the total number of relapses (including the onset attack) divided by the number of years of disease. Two subgroup analyses were performed: A comparison of those with prior exposure to other non- 
steroid maintenance immunotherapies versus treatment naïve patients; and a comparison of adult and paediatric patients.

\section{Results}

Data were obtained from 29 centres in 13 countries - Argentina, Austria, Brazil, France, Germany, Netherlands, India, Italy, Japan, Korea, Switzerland, United Kingdom and United States of America. The total number of MOGAD patients attending all study centres was 875 . RTX was administered to $132 / 875$ (15.1\%). Data on 121 patients were analysed and included in the study. Eleven patients were excluded from the analysis due to incomplete data (6), inadequate treatment protocol (4) and diagnostic uncertainty (1).

\section{Demographics}

Data on 121 patients $(71 / 121,58.7 \%$ female) were analysed (table 1$)$. Median (interquartile range, IQR) age at onset attack was 24.8 (13.1-39.6) years and median age at first RTX infusion was 29.7 (18.2-44.0) years. Race distribution was 103/121 (85.1\%) White, 10/121 $(8.3 \%)$ Asian, $1 / 121(0.8 \%)$ Black, and 7/121 (5.8\%) mixed race. Paediatric patients, meaning those that started RTX before age 18 years, comprised 30/121 (24.7\%). The most common MOGAD phenotypes in adults were relapsing ON $(27 / 91,29.7 \%)$ and relapsing ON with TM $(25 / 91,27.5 \%)$. The most common phenotype in children was ADEM/ADEM-like with relapses $(13 / 30,43.3 \%)$.

\section{Relapses and immunotherapy prior to rituximab}

For all patients, the median (range) disease duration prior to treatment with RTX was 19.1 (1.1-370.7) months. RTX was started after an index attack in 20/121 (16.5\%), and after at least two attacks in 101/121 (83.5\%). The pre-treatment median (IQR) annualized relapse rate 
(ARR) was 1.82 (0.74-3.40) for the 101 relapsing patients. Approximately half of these patients $(54 / 101,53.5 \%)$ were treated with one or more non-steroid immunotherapies before RTX (table 1). These included AZA in 26/101 (25.7\%), MMF in 20/101 (19.8\%) and IVIg in 7/101 (6.9\%). Other immunosuppressive drugs had been used in 13/101 (12.8\%), and MS disease-modifying therapies (MS-DMTs) in 11/101 (10.9\%). There was no standardised 'wash-out' period of prior immunotherapies before starting RTX.

\section{Rituximab dosing}

RTX was administered as a fixed dosing regimen to 115/121 (95.0\%) with an interval of 6 months or less between treatment courses. If only a single treatment course was given, treatment duration was considered as 6 months. A variable dosing interval was used in 6/121 $(5.0 \%)$ on the basis of $\mathrm{CD} 19^{+} \mathrm{B}$-cell count or $\mathrm{CD} 19^{+} / \mathrm{CD} 27^{+}$memory B-cell counts. The most commonly prescribed RTX dose was 1000mg (79/121, 65.3\%). For each treatment course, this was either infused once on day 0 , or twice on day 0 and day 15 . A body surface area adjusted dose of $375 \mathrm{mg} / \mathrm{m}^{2}$, administered weekly for four weeks, was given to $28 / 121$ (23.1\%) and was the usual dose for paediatric patients. A minority of patients received a mix of both dosing regimens $(4 / 121,3.3 \%)$, and in some cases exact dosing was not specified $(10 / 121,8.3 \%)$.

\section{The effect of RTX started after index attack $(n=20)$}

RTX was started after the index attack in 20/121 (16.5\%). Because MOGAD may be monophasic in approximately half of cases (i.e. a significant proportion of these patients may never relapse irrespective of treatment), we analysed this group separately from those with an established relapsing phenotype prior to starting RTX. After a median (IQR) duration on 
RTX of 11.2 (6.3-14.1) months, 14/20 (70\%) remained relapse-free. 11 relapses occurred in $6 / 20(30.0 \%)$ patients, with a median time to first relapse of $2.6(1.3-4.5)$ months.

\section{The effect of RTX started after two or more attacks $(n=101)$}

RTX was started after two or more attacks in 101/121 (83.5\%). The median pre-treatment duration (time from index attack to RTX initiation) was 26.0 (9.8-70.9) months. The median observation period on RTX was 12.1 (6.3-24.9) months, during which 102 relapses occurred in $48 / 101(47.5 \%)$ patients (figure 2). The median time to first relapse was $4.4(1.8-8.5)$ months. Fitting a Poisson regression model showed a 37\% (95\%CI 19-52\%, p <0.001) reduction in relapse rate following treatment with RTX. The Kaplan-Meier estimate of relapse-free survival was 55\% (95\%CI 44-65\%) at 1 year of RTX therapy and $33 \%(95 \% \mathrm{CI}$ $20-46 \%$ ) at 2 years (figure $3 a$ ).

The effect of RTX on median ARR is shown in table 2. For all patients $(n=101)$, the median ARR declined after initiation of RTX from 1.82 to $0.00(\mathrm{p}<0.001$; Wilcoxon signed rank test). Because the calculation of ARR is dependent on the pre- and post-treatment observation periods, we repeated the analysis after excluding patients with short observation periods. In patients with at least 12 months pre-treatment observation $(71 / 101,70.3 \%)$, median ARR declined after initiation of RTX from 1.09 to 0.00 ( $\mathrm{p}<0.001$; Wilcoxon signed rank test). In those with least 12 months post-treatment observation (51/101, 50.5\%), median ARR declined after initiation of RTX from 1.84 to 0.43 ( $\mathrm{p}<0.001$; Wilcoxon signed rank test). Finally, in those with at least 12 months observation both pre- and post-RTX treatment (34/101, 33.7\%), median ARR declined from 1.18 to 0.56 ( $p=0.002$; Wilcoxon signed rank test). 
Early relapses after starting $R T X(n=97)$

Very early relapses after RTX initiation have been described in AQP4-IgG-positive NMOSD and may not signify true RTX failure (see discussion). We therefore re-analysed relapse rates after excluding relapses occurring within 1 month of RTX. The exact dates of 99/102 (97.1\%) relapses were known, and 5/99 (5.1\%) occurred within 1 month of first RTX infusion. Four patients with less than 1 month follow-up on RTX were necessarily excluded from this analysis. With this adjustment, the Poisson regression model showed a 43\% (95\%CI 26$57 \%, \mathrm{p}<0.001)$ reduction in relapse rate. Decline in median ARR was unchanged (table 2).

\section{B-cell depletion $(n=121)$}

A CD $19^{+}$B-cell count $<1 \%$ of circulating lymphocytes is a commonly used indicator of effective B-cell depletion and continued therapeutic action of RTX. ${ }^{30} \mathrm{CD} 19^{+} \mathrm{B}$-cell counts were not systematically acquired in all patients, but were available at the time of $57 / 113$ (50.4\%) relapses occurring on RTX. In 12/57 (21.1\%) relapses the CD19 ${ }^{+}$B-cell count was $\geq 1 \%$, indicating that the B-cell depleting effect of RTX had waned. However, circulating $\mathrm{CD} 19^{+}$B-cells were supressed to $<1 \%$ in $45 / 57$ (78.9\%), indicating disease activity despite effective B-cell depletion. Circulating CD19 ${ }^{+}$B-cells were undetectable at the time of 22/57 $(38.6 \%)$ relapses.

The effect of $R T X$ on treatment naïve patients $(n=47)$ versus those with prior exposure to non-steroid immunotherapies $(n=54)$

To determine if prior exposure to maintenance non-steroid immunotherapies influenced RTX treatment effect, we performed a subgroup analysis comparing relapse rates in treatment naïve patients $(47 / 101,46.5 \%)$ versus those with previous immunotherapy exposure $(54 / 101$, $53.5 \%$, including 11 patients previously treated with MS-DMTs) (table 1). A greater decline 
in median ARR occurred in treatment naïve patients $(\mathrm{p}=0.015$, Mann Whitney $U$ test). The Poisson regression model showed a $63 \%(95 \% \mathrm{CI} 35-79 \%, \mathrm{p}=0.001)$ decline in relapse rate in this group. The Kaplan-Meier estimate of patients treated $1^{\text {st }}$ line with RTX who are relapsefree was 79\% (95\%CI 62-89\%) after 1 year and 55\% (95\%CI 31-73\%) after 2 years (figure $3 b)$.

When RTX was given after other non-steroid immunotherapies, the decline in relapse rate was $26 \%(95 \%$ CI $2-44 \%, p=0.038)$. After 1 and 2 years, $38 \%(95 \%$ CI $25-52 \%)$ and $18 \%$ (95\% CI 7-34\%) were relapse-free respectively (figure 3b). We repeated this analysis after exclusion of the 11 patients with prior exposure to MS-DMTs and the results were similar (table 2), though the observed $25 \%$ decline in relapse rate did not reach statistical significance $(95 \% \mathrm{CI}-3-46 \%, \mathrm{p}=0.077$, Poisson regression model). In the 11 patients exposed to MS-DMTs, the median ARR declined from 2.19 pre-treatment (median observation period 49 months) to 1.79 after initiation of RTX (median observation period 13 months). For the 7 patients with at least 12 months observation pre- and post-RTX treatment, median ARR declined from 1.71 to 0.89 .

The effect of $R T X$ in adults ( $n=71)$ versus children $(n=30)$

RTX was initiated before 18 years of age in 30/121 (24.8\%), all of whom experienced two or more attacks prior to starting RTX. We therefore compared the effect of RTX on relapse rates in 30 children versus $71 / 90(78.0 \%)$ adults, who also had two or more attacks prior to starting RTX (table 2). Median duration on RTX was 12.7 months for adults and 11.8 months for children, in which 31/71 (43.7\%) adults and 17/30 (56.7\%) children relapsed. A Poisson regression model showed a $42 \%(95 \%$ CI $20-59 \%, \mathrm{p}=0.001)$ decline in relapse rate for adults and a $29 \%(95 \% \mathrm{CI}-7-53 \%, \mathrm{p}=0.103)$ decline for children. The proportion of treatment naïve 
patients was $40 / 71(56.3 \%)$ in the adult group versus $7 / 30(23.3 \%)$ in the paediatric group. $\mathrm{CD} 19^{+}$B-cell counts were available for 22/62 (35.5\%) relapses in adults and 30/40 (75.0\%) relapses in children, and were suppressed to $<1 \%$ in $19 / 22(86.4 \%)$ and $21 / 30(70.0 \%)$ respectively.

Use of corticosteroids, steroid-sparing immunotherapies( $n=121)$

Maintenance corticosteroid therapy, defined as daily or alternate day dosing of oral prednisolone (this excludes short-course intravenous methylprednisolone for acute relapses, or single dose pre-medication for RTX infusions), was used in 32/121 (26.4\%) of patients while receiving RTX treatment, of which 17/121 (14.0\%) received continuous treatment, $7 / 121(5.8 \%)$ were treated and then tapered to cessation, and $8(6.6 \%)$ restarted maintenance corticosteroids following a relapse. Maintenance corticosteroids were not used in 78/121 $(64.5 \%)$ and information about steroid use was not available in 11/121 (9.1\%).

Continuation or addition of other steroid-sparing immunotherapies with RTX occurred in 20/121 (16.5\%) patients; MMF (8), IVIg (6), AZA (3), methotrexate (2), IVIg with AZA (1). One patient received low dose IVIg $(0.2 \mathrm{mg} / \mathrm{kg}$ monthly $)$ for RTX-induced hypogammaglobulinaema, and a further patient had recent exposure to alemtuzumab (33 and 21 months pre-RTX). We did not analyse these subgroups due to the low number of patients.

Treatment switches $(n=121)$

22/121 (18.2\%) patients discontinued RTX, due to relapses $(16 / 22,72.7 \%)$ or de-escalation of immunotherapy $(5 / 22,22.7 \%)$. These patients commenced a variety of alternative immunomodulatory therapies (e.g. MMF [n=4], tocilizumab [n=3], AZA [n=3], IVIg [n=4] 
and other multi-drug regimens). One patient stopped RTX due to an infection (cryptococcal meningoencephalitis).

\section{EDSS and visual disability $(n=121)$}

To determine if EDSS was affected by RTX treatment we compared EDSS at RTX initiation and at last review or on switching to an alternative immunotherapy. EDSS data were available for $97 / 121(80.2 \%)$ patients, but scores were not assessed at defined time points with respect to relapses. Median (IQR) EDSS score improved from 3.0 (2.0-3.5) at RTX initiation to $2.0(1.0-3.0)$ at follow-up $(\mathrm{z}=3.36, \mathrm{p}=0.001$; Wilcoxon signed rank test).

Visual disability was similarly analysed. VFS scores were calculated on 41/121 (33.9\%) patients with sufficient data, of whom 21/41 (51.2\%) experienced ON during the RTX treatment period. Overall, there was no change in median VFS score, which was $2(1-4)$ on RTX initiation and $2(0-4)$ at follow-up ( $\mathrm{t}=1.99, \mathrm{p}=0.03$; paired $\mathrm{t}$-test). For patients with at least one episode of ON during RTX treatment, VFS scores improved in 8/21 (38.1\%), remained stable in 10/21 (47.6\%) and worsened in 3/21 (14.3\%).

\section{Tolerance and adverse events}

We did not systematically acquire data on tolerance and adverse events in this study. However, the following serious adverse events were reported: anaphylactoid infusion reaction (1), hypogammaglobulinaemia (1) and cryptococcal meningoencephalitis (1). No patients died during treatment with RTX.

\section{Discussion}


This is the first study to examine RTX efficacy in a large cohort of MOGAD patients. RTX treatment led to a $37 \%$ decline in relapse rate, and after 2 years of treatment, $33 \%$ of patients remained relapse-free. This is a less beneficial effect than has been observed with anti-CD20 B-cell depletion in similar neuroinflammatory disorders - MS and NMOSD. Where circulating $\mathrm{CD} 19^{+}$B-cell counts were available, $79 \%$ of relapses occurred despite robust Bcell depletion. The greatest treatment effect $(63 \%$ decline in relapse rate) was observed in patients who received RTX as a first line maintenance immunotherapy. Patients with prior exposure to other steroid-sparing medications (usually AZA and MMF), experienced only a $25 \%$ decline in relapses. Separate analyses of adults and children suggested a better response in adult patients ( $42 \%$ versus $29 \%$ reduction in relapse rates).

The true benefit of RTX in MOGAD may be even less than that observed in this study, when one considers the potential influence of regression to the mean. This refers to the tendency of a group to return to the average, rather than to sustain an above average relapse rate. To assess the quantitative importance of the regression to the mean phenomenon, it is important to understand the behaviour of historic cohorts. For example, in randomised controlled trials in relapsing MS, regression to the mean may explain as much as $40 \%$ of the reduction of relapse rate, which is observed in both treatment and placebo arms. ${ }^{31}$ In MOGAD, estimates of median ARR in untreated and immunosuppressed cohorts have varied greatly and have been prejudiced by both testing bias and short observation periods. It is therefore difficult to quantify the effect of regression to the mean in studies of treatment effect. However, in this study, where the observed treatment effect is relatively small, it is particularly important to consider regression to the mean when drawing conclusions. 
Previous studies of treatment effect in MOGAD included small numbers of RTX-treated patients. They also observed a reduction of relapse rate following initiation of RTX, but described frequent early relapses: A German study reported that 6/9 patients relapsed during treatment with RTX. ${ }^{9}$ An Australasian study included six rituximab-treated patients, of whom one patient experienced two relapses despite B-cell depletion. ${ }^{15}$ Finally, in a European paediatric study, 6/9 RTX-treated children relapsed and one child had a severe lifethreatening relapse despite B-cell depletion. Of the three children that did not relapse, two were additionally receiving maintenance IVIg. ${ }^{14}$

It is not clear why RTX appears less effective for MOGAD as compared to MS and NMOSD. Phase I and II randomised controlled trials of RTX in relapsing MS met their primary endpoints, and the pivotal phase III trials of ocrelizumab, a similar anti-CD20 therapy, versus interferon beta-1a, reported a 46-47\% lower relapse rate with ocrelizumab, establishing Bcell depletion as important therapeutic strategy for MS. ${ }^{16-18}$ Likewise, retrospective studies of RTX in NMOSD have consistently reported high response rates with substantial reductions in ARR. ${ }^{20-29}$ The largest meta-analysis of RTX in predominantly AQP4-IgG positive NMOSD included 46 studies of 438 patients and calculated a $79 \%$ reduction in relapse rate. ${ }^{28}$ RTX has therefore become a dependable maintenance therapy for AQP4-IgG positive NMOSD in countries where it is readily available and affordable.

One explanation for apparent lack of efficacy is that this study has selected out a subgroup of MOG-IgG positive patients with highly active and treatment-refractory disease. Only $15.1 \%$ of all MOG-IgG patients that attend the participating centres have been treated with RTX. This figure probably over-estimates RTX usage in MOGAD. Firstly, because testing for MOG-IgG is not widely available in all countries, and monophasic or infrequently relapsing 
patients are least likely to be tested for MOG-IgG, there is a bias to diagnosing more active cases. Secondly, many authors in this study run specialist tertiary or quaternary referral clinics and will therefore tend to encounter the more active or treatment-refractory cases. High disease activity in this study population is evidenced by the relatively high pretreatment median ARR of 1.82, or 1.09 after excluding patients with less than 12 months pretreatment observation. The latter is a more reliable estimate of the true ARR in this group, and is nonetheless considerably higher than the median ARR of $0.20-0.38$ reported for UK and French incident cohorts (patients diagnosed after their index attack).,8

Selection of increasingly treatment-refractory patients may also explain why treatment naïve patients appeared to respond better to RTX than those who had failed treatment with other steroid-sparing immunosuppressive medications. Although pre-RTX ARR was higher (2.36 versus 1.45) in the treatment naïve group, it is doubtful that this accounts fully for the improved response observed. A treatment paradox, in which higher relapse rates and poorer outcomes are seen in the group receiving more therapy, has been observed in other neuroinflammatory disorders and reflects the a priori threshold for initiating such treatments. $^{32,33}$ Similarly, the apparently poor efficacy of RTX observed in retrospective systematic reviews of acetylcholine receptor antibody positive myasthaenia gravis (AChRMG), has been postulated to result from the inclusion of many 'burnt out', treatmentrefractory cases..$^{34,35}$

We explored whether very early relapses may be accounting for apparently poor efficacy of RTX by excluding those occurring within a month of RTX initiation (5\% of relapses in this study). This increased the calculated decline in relapse rate, but only slightly, from $37 \%$ to $43 \%$. The validity of this adjustment is uncertain in MOGAD, but stems from experience in 
AQP4-IgG-positive NMOSD, where a lag time of 3-4 weeks in terms of relapse-preventing effect has been described in several studies, despite achievement of circulating B-cell depletion within hours to days of first RTX infusion. ${ }^{24,36}$ An increased relapse risk during this lag time has even been observed by some, possibly due to elevation of systemic B-cell activating factor, which could induce antibody secretion by CD20- plasma cells. ${ }^{37,38}$ For these reasons many neurologists treating NMOSD do not consider very early relapses to reflect truly RTX-refractory disease. ${ }^{30}$

Importantly, it may be that circulating $\mathrm{CD} 20^{+}$B-cell depletion with RTX is truly less efficacious for MOGAD than for MS and AQP4-IgG-positive NMOSD. Despite their overlapping clinical phenotypes, many differences have been identified between the immunopathogenic mechanisms underpinning these disorders. It is also unclear, for example whether the efficacy of RTX in AQP4-IgG-positive NMOSD is due to suppression of antibody production, modulation of downstream antibody-mediated pathways, or a combination of mechanisms. Data on longitudinal MOG-IgG titres were not collected as part of this study, but may be informative in future studies of treatment effect, as loss of seropositivity appears more common in MOGAD as compared to AQP4-IgG-positive NMOSD, and seems to correlate (though not completely) with lower relapse risk, at least over short-term follow-up. ${ }^{6-9,39,40}$ It could be that antibody production by CD20- plasma cells, or by B-cells sequestered in secondary lymphoid tissue are responsible for RTX-refractory MOGAD disease.

Both AQP4-IgG and MOG-IgG are of the IgG1 immunoglobulin subclass, but the evidence for a direct pathogenic effect of MOG-IgG in the CNS is less certain than for AQP4-IgG. Spadaro et al. (2018) showed that the pathogenicity of human MOG-IgG is dependent on 
interactions with T-cells: In synergy with myelin basic protein-specific T-cells, MOG-IgG mediated MS type II pathology, and together with MOG-specific T-cells, it enhanced T-cell infiltration. $^{41}$ Others have shown, using murine models, that MOG-IgG may cause complement-mediated demyelination. ${ }^{42,43}$

It is also interesting that the children in this study experienced only a $29 \%$ reduction in relapse rates on RTX (which did not reach statistical significance, $\mathrm{p}=0.103$ ), as compared to the $42 \%$ decline observed in adults. Children comprised only a quarter of the study population, and one of the 30 patients had 9 relapses on RTX, which may have skewed the data. A greater proportion of adult patients ( $56 \%$ versus $23 \%)$ were treatment naïve prior to RTX, which may have affected the observed efficacy, as described above. Finally, of all relapses that were known to have occurred in the context of inadequate B-cell depletion (CD19+ B-cell count $\geq 1 \%), 9 / 12(75 \%)$ occurred in children. This could suggest that closer monitoring of B-cell counts was warranted in these children, rather than a true failure of Bcell depletion itself. Nevertheless, the apparently different response to RTX of adults and children with MOGAD merits further study.

Although $45 \%$ of the cohort experienced at least one relapse during RTX therapy, only $18 \%$ of patients switched to an alternative immunotherapy. This could be because on-treatment relapses were mild or responded well to acute therapy. The EDSS and VFS data, which show a trend towards improving disability and stable visual function, may support this. However, these data were incomplete in our study; EDSS and VFS scores were available for $80 \%$ and $35 \%$ of the cohort respectively, and were not calculated at designated time points with respect to prior attacks. Immunosuppressive therapy is most often commenced or changed in the immediate aftermath of an acute relapse, while recovery continues and EDSS has not yet 
stabilised. The EDSS score is also heavily weighted on motor and ambulatory performance and may therefore be poorly sensitive to accrual of visual disability and bladder and bowel dysfunction, which are thought to be preferentially affected in MOGAD. ${ }^{7}$

Another reason for continuing RTX in the face of ongoing relapses may be the limited options and evidence base for third-line therapies in MOGAD. A retrospective study that compared treatment effects in children with relapsing MOGAD suggested that regular IVIg infusions may be the most effective treatment, but this needs further study. ${ }^{14}$ Other monoclonal antibody therapies used for RTX-refractory AQP4-IgG positive NMOSD, such as tocilizumab (anti-interleukin-6 receptor), have been used infrequently in MOGAD. Finally, reports of severe flares AQP4-IgG positive NMOSD following administration of MS disease-modifying therapies, including beta-interferons, fingolimod, natalizumab and alemtuzumab, have prompted avoidance of such medications once a diagnosis of antibodymediated CNS inflammation is established. In this study, the 11 patients with prior exposure to MS therapies did have a relatively high pre-RTX median ARR of 2.19 , but we did not specifically examine the temporal relationship between relapses and MS therapies.

The limitations of this study include its retrospective design and the necessary inclusion of many patients with relatively short on-treatment follow-up periods. The latter will bias analysis of ARRs, often used in this type of study, and this is why we used the Poisson regression model as our primary statistical model, to provide a more meaningful analysis of treatment effect with such variable follow-up periods. Another limitation is the baseline heterogeneity of the cohort in terms of patient ages, MOGAD phenotypes and prior drug exposure. Furthermore, many patients received concomitant corticosteroid treatment at changing doses, there was no standardised washout from prior steroid-sparing medications 
and a minority of patients continued these treatments alongside RTX therapy. These factors influence relapse risk and so introduce a bias to this study, but are inherent to real-world, retrospective studies of this nature. The inclusion of all cases improves the generalisability of this study and reflects the challenges of managing this rare and unpredictable disorder with the potential to cause permanent neurological disability. Prospective randomised controlled trials, in which prior and concomitant drug therapy can be controlled, and where B-cell counts and MOG-IgG titres can be systematically acquired, will better define the role of RTX in the treatment of MOGAD.

\section{Conclusion}

An overall decline in MOGAD relapse rates was observed after initiation of RTX. However, when compared to similar studies in MS and AQP4-IgG positive NMOSD, RTX appeared less effective. Furthermore, many relapses in this study occurred despite robust circulating Bcell depletion. This study highlights the need to understand the immunopathogenic mechanisms underlying MOGAD and the need for prospective head-to-head comparative studies of treatment effect, which include longitudinal antibody testing. At present, there is enough clinical equipoise to justify randomised placebo-controlled trials in MOGAD, which should provide more robust answers to important management questions. 
Figure 1: Flow diagram demonstrating data analysis

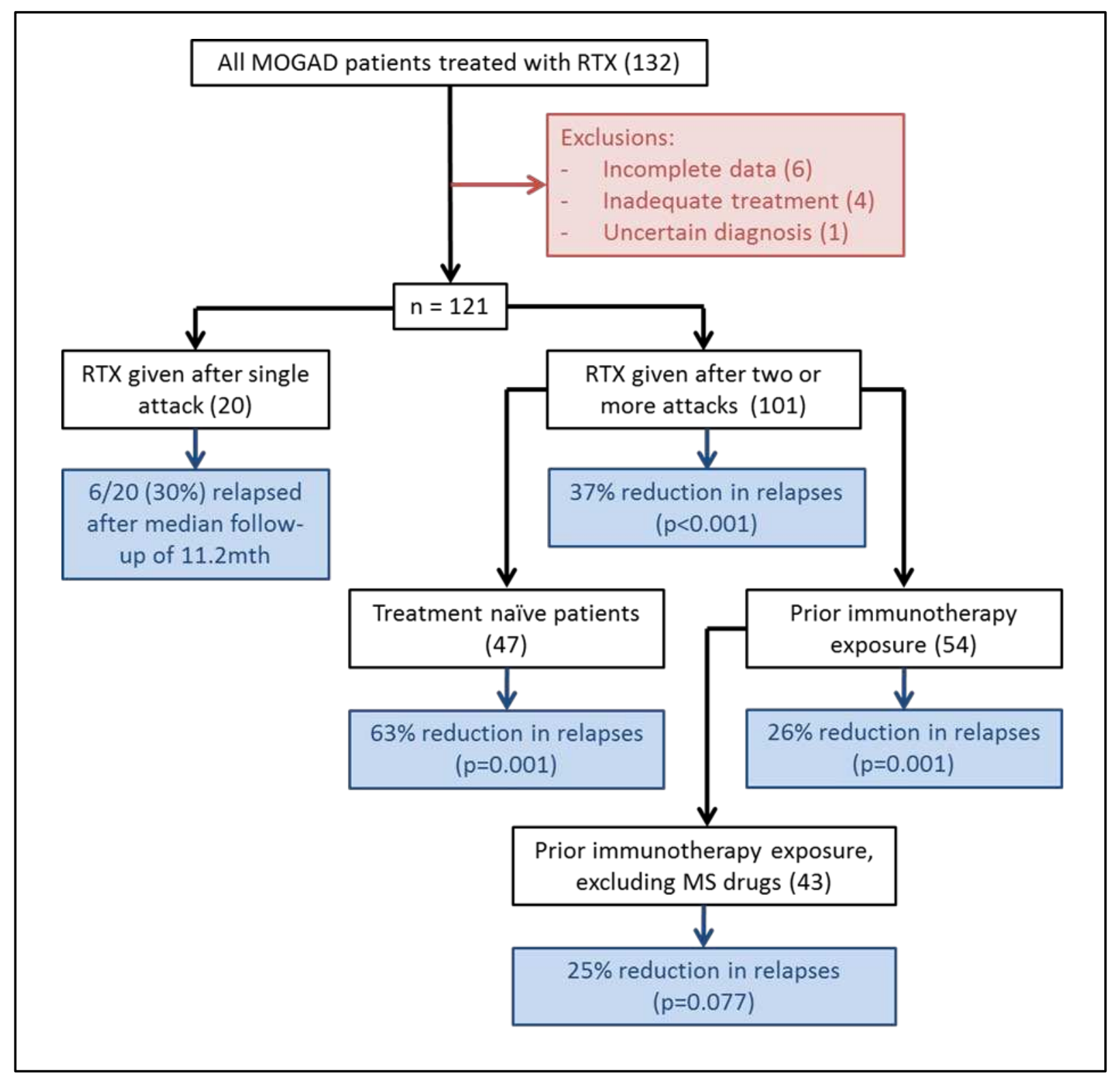


Table 1: Cohort demographics, clinical phenotypes and previous immunotherapies

\begin{tabular}{|c|c|c|c|c|c|}
\hline & Whole cohort & Adults & Children & $\begin{array}{l}\text { Single attack } \\
\text { pre-RTX } \\
\text { (all ages) }\end{array}$ & $\begin{array}{l}\text { Multiple } \\
\text { attacks } \\
\text { pre-RTX } \\
\text { (all ages) } \\
\end{array}$ \\
\hline Patients, $\mathrm{n}$ & 121 & 91 & 30 & 20 & 101 \\
\hline Female, n (\%) & $71(58.7)$ & $56(61.5)$ & $15(50.0)$ & $14(70.0)$ & $57(56.4)$ \\
\hline White, $\mathrm{n}(\%)$ & $103(85.1)$ & $84(69.4)$ & $19(63.3)$ & $19(95.0)$ & $84(83.2)$ \\
\hline Median (IQR) onset age, yrs & $24.8(13.1-39.6)$ & $33.0(22.7-43.5)$ & $7.6(4.0-9.9)$ & $31.9(22.5-40.9)$ & $23.2(10.9-38.8)$ \\
\hline Median (IQR) RTX start age, yrs & $29.7(18.2-44.0)$ & $37.7(25.5-48.5)$ & $11.7(8.3-14.0)$ & $32.1(22.7-41.5)$ & $27.2(16.4-44.1)$ \\
\hline Disease onset $<18$ years, $\mathrm{n}(\%)$ & $39(32.2)$ & $9(9.9)$ & $30(100)$ & 0 & $39(38.6)$ \\
\hline RTX initiation <18years, $\mathrm{n}(\%)$ & $30(24.7)$ & 0 & $30(100)$ & 0 & $30(29.7)$ \\
\hline $\begin{array}{l}\text { Median (IQR) disease duration } \\
\text { pre-RTX, mths }\end{array}$ & $19.1(5.9-55.0)$ & $12.8(5.0-49.1)$ & $33.0(16.3-69.6)$ & $3.3(1.7-5.1)$ & $26.0(9.8-70.9)$ \\
\hline $\begin{array}{l}\text { Phenotypes, } \mathbf{n}(\%) \text { : } \\
\text { Single attack } \\
-\quad \text { ON } \\
-\quad \text { TM } \\
-\quad \text { ON + TM } \\
\text { Relapsing } \\
-\quad \text { ON } \\
-\quad \text { TM } \\
-\quad \text { ON + TM } \\
-\quad \text { ADEM/ADEM-like } \\
-\quad \text { Other / brain } \\
\\
\quad \text { involvement } \\
\end{array}$ & $\begin{array}{c}20(16.5) \\
6(5.0) \\
9(7.4) \\
5(4.1) \\
101(83.5) \\
29(24.0) \\
6(5.0) \\
29(24.0) \\
1512.4) \\
22(18.2)\end{array}$ & $\begin{array}{c}20(21.9) \\
6(6.6) \\
9(9.9) \\
5(5.5) \\
71(78.0) \\
27(29.7) \\
5(5.5) \\
25(27.5) \\
2(2.2) \\
12(13.2)\end{array}$ & $\begin{array}{l}0 \\
\\
30(100) \\
2(6.7) \\
1(3.3) \\
4(13.3) \\
13(43.3) \\
10(33.3)\end{array}$ & $\begin{array}{l}20(100) \\
6(30.0) \\
9(45.0) \\
5(25.0)\end{array}$ & $\begin{array}{l}101(100) \\
29(28.7) \\
6(5.9) \\
29(28.7) \\
15(14.9) \\
22(21.8)\end{array}$ \\
\hline Median pre-RTX ARR & 2.25 & 2.34 & 1.64 & & 1.82 \\
\hline $\begin{array}{ll}\text { No. of steroid-sparing ITs prior } \\
\text { to RTX, } \mathrm{n}(\%) \text { : } \\
\begin{array}{rc}- & 0 \\
- & 1 \\
- & 2 \\
- & 3+ \\
\end{array}\end{array}$ & $\begin{array}{l}66(54.5) \\
35(28.9) \\
13(10.7) \\
7(5.8) \\
\end{array}$ & $\begin{array}{l}59(64.8) \\
19(20.9) \\
9(9.9) \\
4(4.4) \\
\end{array}$ & $\begin{array}{l}7(23.3) \\
16(53.3) \\
4(13.3) \\
3(10.0) \\
\end{array}$ & $\begin{array}{l}19(95.0) \\
1(5.0) \\
0 \\
0 \\
\end{array}$ & $\begin{array}{l}47(46.5) \\
34(33.7) \\
13(12.9) \\
7(6.9) \\
\end{array}$ \\
\hline \begin{tabular}{cl}
\multicolumn{2}{l}{ Types of prior steroid-sparing } \\
ITs prior to RTX, $\mathrm{n}\left(\%^{*}\right)$ \\
- & None \\
- & AZA \\
- & MMF \\
- & Other IS* \\
- & IVIg \\
- & Maintenance PLEX \\
- & Any MS-DMT \\
- & Injectable MS-DMT \\
- & Oral/infusible MS- \\
& DMT
\end{tabular} & $\begin{array}{l}66(54.5) \\
27(22.3) \\
20(16.5) \\
13(10.7) \\
7(5.8) \\
2(1.7) \\
11(9.1) \\
9(7.4) \\
5(4.1)\end{array}$ & $\begin{array}{l}59(64.8) \\
13(14.3) \\
14(15.4) \\
9(9.9) \\
1(1.1) \\
1(1.1) \\
8(8.8) \\
6(6.6) \\
5(5.5)\end{array}$ & $\begin{array}{l}7(23.3) \\
14(46.7) \\
6(20.0) \\
4(13.3) \\
6(20.0) \\
1(3.3) \\
3(10.0) \\
3(10.0) \\
0\end{array}$ & $\begin{array}{l}19(95.0) \\
1(5.0) \\
0 \\
0 \\
0 \\
0 \\
0 \\
0 \\
0\end{array}$ & $\begin{array}{l}47(46.5) \\
26(25.7) \\
20(19.8) \\
13(12.9) \\
7(6.9) \\
2(2.0) \\
11(10.9) \\
9(8.9) \\
5(5.0)\end{array}$ \\
\hline
\end{tabular}


Figure 2: MOGAD relapses occurring before and after treatment with rituximab

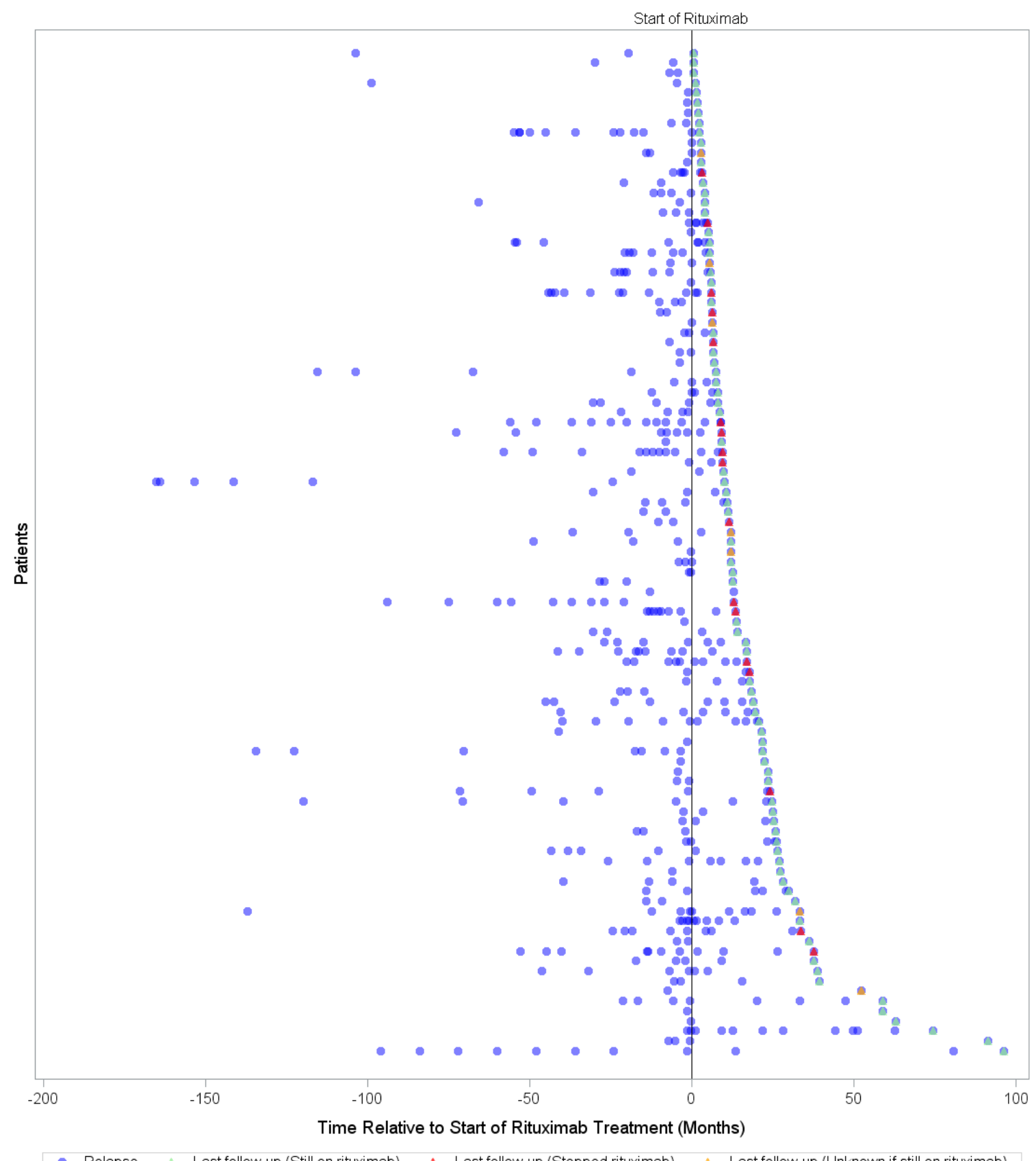


Table 2: The effect of rituximab on relapse rates in MOGAD

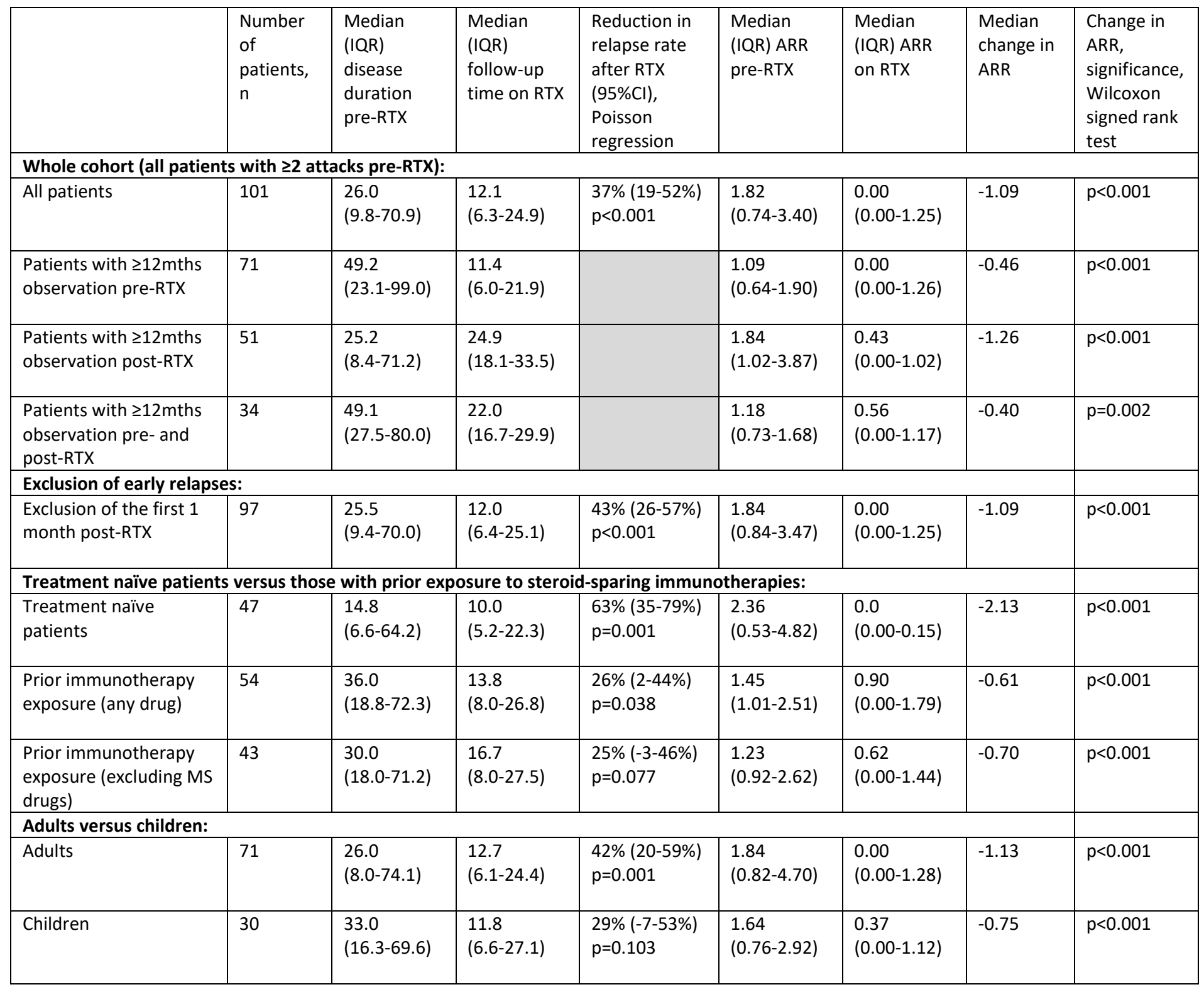


Figure 3a: Kaplan-Meier plot of relapse-free survival following initiation of rituximab.

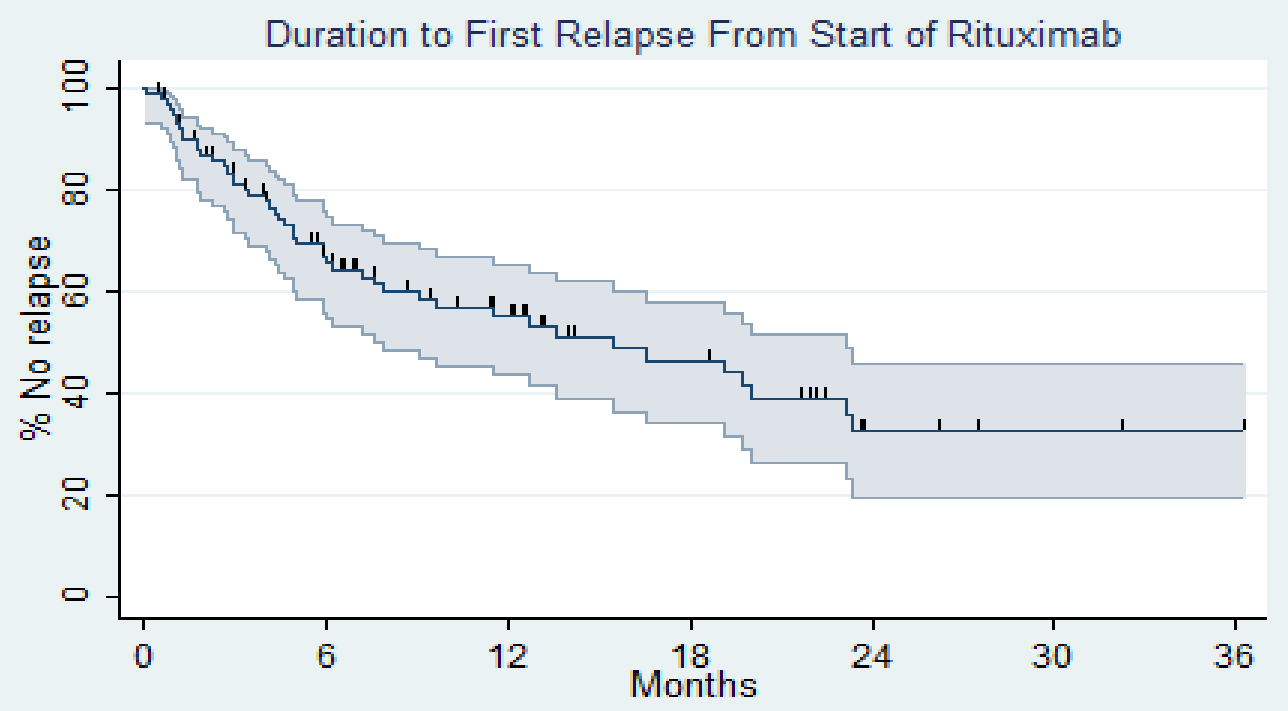

$\mathrm{N}$ at risk 101

\begin{tabular}{|ccccc|}
53 & 30 & 20 & 8 & 6 \\
\hline & $95 \% \mathrm{Cl}$ & &
\end{tabular}

5

Figure 3b: Kaplan-Meier plot of relapse-free survival following initiation of rituximab, comparing treatment-naïve patients and those with previous exposure to non-steroid immunotherapies

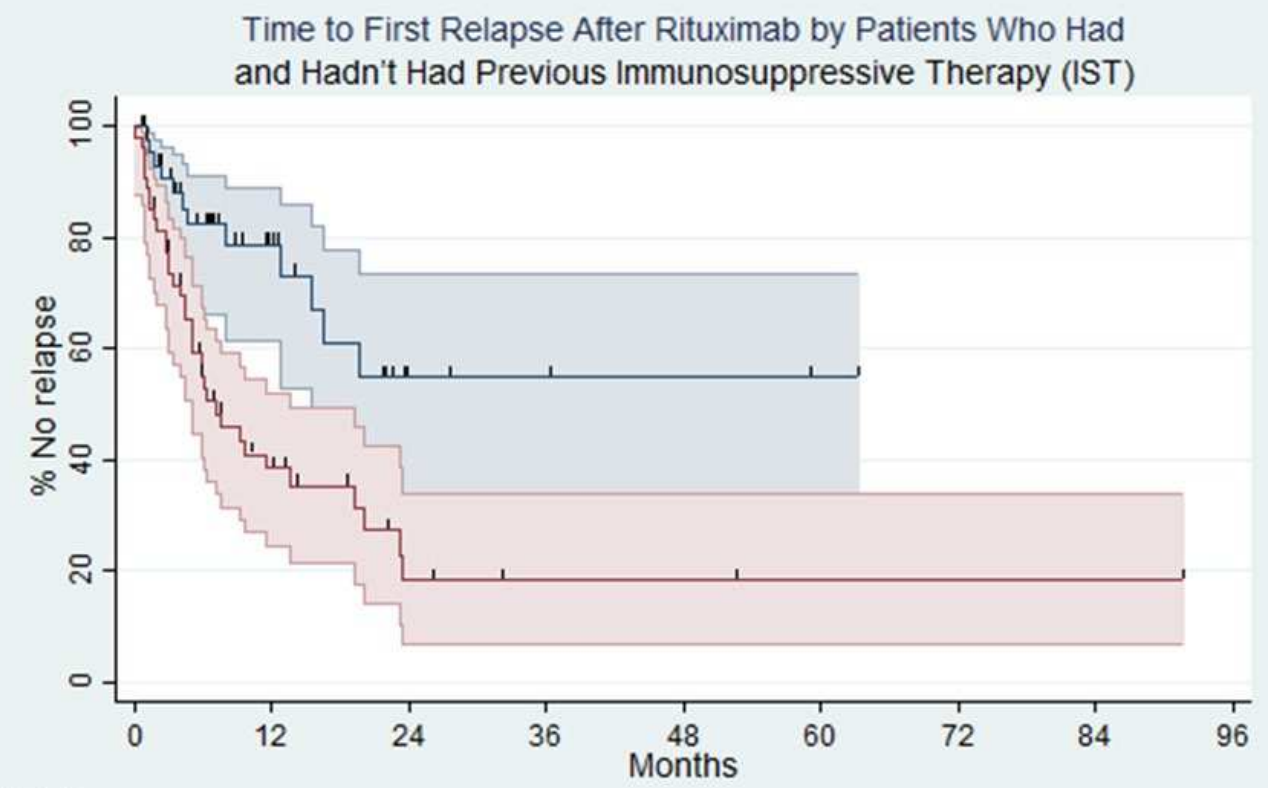

$\mathrm{N}$ at risk

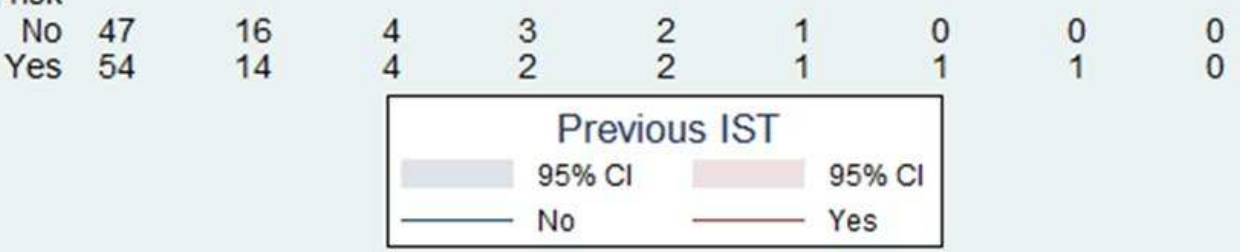




\section{References}

1. O'Connor K, McLaughlin KA, De Jager PL, et al. Self-antigen tetramers discriminate between myelin autoantibodies to native or denatured protein. Nat Med 2007;13(2):211-217.

2. Mader S, Gredler V, Schanda K, et al. Complement activating antibodies to myelin oligodendrocyte glycoprotein in neuromyelitis optica and related disorders. $J$ Neuroinflammation 2011;8:184.

3. Kitley J, Woodhall M, Waters P, et al. Myelin-oligodendrocyte glycoprotein antibodies in adults with a neuromyelitis optica phenotype. Neurology 2012;79(12):1273-1277.

4. Jarius S, Paul F, Aktas O, et al. MOG encephalomyelitis: international recommendations on diagnosis and antibody testing. J Neuroinflammation 2018;15(1):134.

5. Hamid SHM, Whittam D, Saviour M, et al. Seizures and encephalitis in myelin oligodendrocyte glycoprotein IgG disease vs aquaporin 4 IgG disease. JAMA Neurol $2018 ; 75(1) 65-71$

6. Lopez-Chiriboga AS, Majed M, Fryer J, et al. Association of MOG-IgG serostatus with relapse after acute disseminated encephalomyelitis and proposed diagnostic criteria for MOG-IgG-associated disorders. JAMA Neurol 2018;75(11):1355-1363.

7. Jurynczyk M, Messina S, Woodhall MR, et al. Clinical presentation and prognosis in MOG-antibody disease: A UK study. Brain 2017;140(12):3128-3138.

8. Cobo-Calvo A, Ruiz A, Maillart E, et al. Clinical spectrum and prognostic value of CNS MOG autoimmunity in adults: The MOGADOR study. Neurology 2018;90(21):e1858-e1869. 
9. Jarius S, Ruprecht K, Kleiter I, et al. MOG-IgG in NMO and related disorders: a multicenter study of 50 patients. Part 2: Epidemiology, clinical presentation, radiological and laboratory features, treatment responses, and long-term outcome. $J$ Neuroinflammation 2016;13(1):280.

10. Hamid SHM, Whittam D, Mutch K, et al. What proportion of AQP4-IgG-negative NMO spectrum disorder patients are MOG-IgG positive? A cross sectional study of 132 patients. $J$ Neurol 2017;264(10):2088-2094.

11. Palace J, Leite I, Jacob A. A practical guide to the management of neuromyelitis optica. Pract Neurol 2012;12(4):205-205.

12. Kimbrough DJ, Fujihara K, Jacob A, et al. Treatment of neuromyelitis optica: review and recommendations. Mult Scler Relat Disord 2012;1(4):180-187.

13. Jurynczyk M, Jacob A, Fujihara K, Palace J. Myelin oligodendrocyte glycoprotein (MOG) antibody-associated disease: practical considerations. Pract Neurol 2018 [Epub ahead of print]

14. Hachohen Y, Wong YY, Lechner C, et al. Disease course and treatment responses in children with relapsing myelin oligodendrocyte glycoprotein antibody-associated disease. JAMA Neurol 2018;75(4):478-487.

15. Ramanathan S, Mohammad S, Tantsis E, et al. Clinical course, therapeutic responses and outcomes in relapsing MOG antibody-associated demyelination. $J$ Neurol Neurosurg Psychiatry 2018;89(2):127-137.

16. Bar-Or A, Calabresi PA, Arnold D, et al. Rituximab in relapsing-remitting multiple sclerosis: a 72-week, open-label, phase I trial. Ann Neurol 2008;63(3):395-400.

17. Hauser SL, Waubant E, Arnold DL, et al. B-cell depletion with rituximab in relapsing-remitting multiple sclerosis. N Engl J Med 2008;358(7):676-688. 
18. Hauser SL, Bar-Or A, Comi G, et al. Ocrelizumab versus interferon beta-1a in relapsing multiple sclerosis. $N$ Engl J Med 2017;376(3):221-234.

19. Montalban X, Hauser SL, Kappos L, et al. Ocrelizumab versus placebo in primary progressive multiple sclerosis. $N$ Engl J Med 2017;376(3):209-220.

20. Cree BA, Lamb S, Morgan K, Chen A, Waubant E, Genain C. An open label study of the effects of rituximab in neuromyelitis optica. Neurology 2005;64(7):1270-1272.

21. Jacob A, Weinshenker BG, Violich I, et al. Treatment of neuromyelitis optica with rituximab: retrospective analysis of 25 patients. Arch Neurol 2008;65(11):1443-1448.

22. Pellkofer HL, Krumbholz M, Berthele A, et al. Long-term follow-up of patients with neuromyelitis optica after repeated therapy with rituximab. Neurology 2011;76:13101315.

23. Mealy MA, Wingerchuk DM, Palace J, et al. Comparison of relapse and treatment failure rates among patients with neuromyelitis optica: multicenter study of treatment efficacy. JAMA Neurol 2014;71:324-330.

24. Kim SH, Kim W, Li XF, et al. Repeated treatment with rituximab based on the assessment of peripheral circulating memory B-cells in patients with relapsing neuromyelitis optica over 2 years. Arch Neurol 2011;68:1412-1419.

25. Kim SH, Huh SY, Lee SJ, et al. A 5-year follow-up of rituximab treatment in patients with neuromyelitis optica spectrum disorder. JAMA Neurol 2013;70:1110-1117.

26. Kim SH, Jeong IH, Hyun JW, et al. Treatment outcomes with rituximab in 100 patients with neuromyelitis optica: Influence of FCGR3A polymorphisms on the therapeutic response to rituximab. JAMA Neurol 2015;72(9):989-995.

27. Radaelli M, Moiola L, Sangalli F, et al. Neuromyelitis optica spectrum disorders: Long-term safety and efficacy of rituximab in Caucasian patients. Mult Scler 2016;22(4):511-519. 
28. Damato V, Evoli A, Iorio R. Efficacy and safety of rituximab therapy in neuromyelitis optica spectrum disorders: A systematic review and meta-analysis. JAMA Neurol 2016; 73(11):1342-1348.

29. Cohen M, Romero G, Bas J, et al. Monitoring CD27+ memory B-cells in neuromyelitis optica spectrum disorders patients treated with rituximab: results from a bicentric study. J Neurol Sci 2017;373:335-338.

30. Kimbrough DJ, Fujihara K, Jacob A, et al. Treatment of neuromyelitis optica: review and recommendations. Mult Scler Relat Disord 2012;1(4):180-187.

31. Martínez-Yélamos S, Martínez-Yélamos A, Martín Ozaeta G, Casado V, Camona O, Arbizu T. Regression to the mean in multiple sclerosis. Mult Scler 2006;16(6):826829.

32. Titulaer MJ, McCracken L, Gabilondo I, et al. Treatment and prognostic factors for long-term outcome in patients with anti-NMDA receptor encephalitis. Lancet Neurol 2013;12(2):157-165.

33. Deiva K, Absoud M, Hemingway C, et al. UK Childhood Inflammatory Demyelination (UK-CID) Study and French Kidbiosep Study. Acute idiopathic transverse myelitis in children. Neurology 2015;84(4):341-349.

34. Iorio R, Damato V, Alboini PE, et al. Efficacy and safety of rituximab for myasthenia gravis: a systematic review and meta-analysis. J Neurol 2015;262:1115-1119.

35. Tandan R, Hehir MK, Waheed W, et al. Rituximab treatment of myasthenia gravis: a systematic review. Muscle Nerve 2017;56:185-96.

36. Lindsey JW, Meulmester KM, Brod SA, et al. Variable results after rituximab in neuromyelitis optica. J Neurol Sci 2012;317(1-2):103-105.

37. Perumal JS, Kister I, Howard J, et al. Disease exacerbation after rituximab induction in neuromyelitis optica. Neurol Neuroimmunol Neuroinflamm 2015;2(1):e61. 
38. Nakashima I, Takahashi T, Cree BA, et al. Transient increases in anti-aquaporin-4 antibody titers following rituximab treatment in neuromyelitis optica, in association with elevated serum BAFF levels. J Clin Neurosci 2011;18(7):997-998.

39. Hyun JW, Woodhall MR, Kim SH, et al. Longitudinal analysis of myelin oligodendrocyte glycoprotein antibodies in CNS inflammatory diseases. $J$ Neurol Neurosurg Psychiatry 2017;88(10):811-817.

40. Oliveira LM, Apóstolos-Pereira SL, Pitombeira MS, Bruel Torretta PH, Callegaro D, Sato DK. Persistent MOG-IgG positivity is a predictor of recurrence in MOG-IgGassociated optic neuritis, encephalitis and myelitis. Mult Scler 2018 [Epub ahead of print].

41. Spadaro M, Winklmeier S, Beltrán E, et al. Pathogenicity of human antibodies against myelin oligodendrocyte glycoprotein. Ann Neurol 2018;84(2):315-318.

42. Peschl P, Schanda K, Zeka B, et al. Human antibodies against the myelin oligodendrocyte glycoprotein can cause complement-dependent demyelination. $J$ Neuroinflammation 2017;14(1):208.

43. Fang L, Kang X, Wang Z, et al. Myelin oligodendrocyte glycoprotein-IgG contributes to oligodendrocytopathy in the presence of complement, distinct from astrocytopathy induced by AQP4-IgG. Neurosci Bull 2019 [Epub ahead of print]. 